\title{
NONDESTRUCTIVE TESTING OF CERAMIC HIP JOINT IMPLANTS WITH LASER SPOT THERMOGRAPHY
}

The paper presents an application of laser spot thermography for damage detection in ceramic samples with surface breaking cracks. The measurement technique is an active thermographic approach based on an external heat delivery to a test sample, by means of a laser pulse, and signal acquisition by an infrared camera. Damage detection is based on the analysis of surface temperature distribution near the exciting laser spot. The technique is nondestructive, non-contact and allows for full-field measurements. Surface breaking cracks are a very common type of damage in ceramic materials that are introduced in the manufacturing process or during the service period. This paper briefly discusses theoretical background of laser spot thermography, describes the experimental test rig and signal processing methods involved. Damage detection results obtained with laser spot thermography are compared with reference measurements obtained with vibrothermography. This is a different modality of active thermography, that has been previously proven effective for this type of damage. We demonstrate that both measurement techniques can be effectively used for damage detection and quality control applications of ceramic materials.

Keywords: laser spot thermography, damage detection, ceramic materials, hip joint implants

\section{Introduction}

Nondestructive testing (NDT) is an integral part of modern engineering applications. It is utilized at every stage of product development, from the manufacturing to the service period. There are many well established nondestructive testing methods like: visual inspection, electromagnetic testing, magnetic testing, ultrasonic testing, liquid penetrant testing, thermography or x-ray [1-2]. Among them thermographic methods become more and more popular due their unique features and versatility that make them very attractive in the fields like aerospace, civil engineering, automotive or biomedical [2-3].

The focus of this paper is on damage detection in ceramic materials used in medical applications. Ceramic materials are brittle and prone to internal and surface cracking that can arise due to a number of factors including the manufacturing process (sintering, green processing, impurities in the powder) or during the service period [4-6]. Common techniques utilized for identification of surface breaking cracks in ceramics are visual testing and liquid penetrant testing. The former may eliminate only the large evident flaws, while the latter offers much higher detection sensitivity. The principle of liquid penetrant testing is that the liquid penetrant is drawn into the surface-breaking crack by capillary action and excess surface penetrant is then removed; a developer (typically a dry powder) is then applied to the surface, to draw out the penetrant in the crack and pro- duce a surface indication. Cracks as narrow as 150 nanometers can be detected. The indications produced are much broader than the actual flaw and are therefore more easily visible $[7,8]$. The detection capability of this technique is very good, there are however several drawbacks. It is relatively expensive, as it involves highly qualified inspection personnel, and it is time consuming, as there is a need to apply the penetrant and remove it after testing. Therefore, there is a demand for testing methods that offer shorter inspection times and can be automated. Thermographic Nondestructive Testing (TNDT) may provide a viable alternative, by offering faster, more economical, non-contact and nondestructive damage detection [2,9-12].

This paper presents an application and comparison of two TNDT techniques, namely the laser spot thermography and vibrothermography, for damage detection in ceramic components with surface breaking cracks. The paper provides a theoretical description of both considered TNDT testing methods, discusses their experimental arrangements and necessary signal processing. Finally, results obtained from laser spot thermography and vibrothermography are presented and compared.

\section{Problem statement}

Ceramic components mostly fail due to internal flaws, like pores, cracks or inclusions, which are propagating in an 


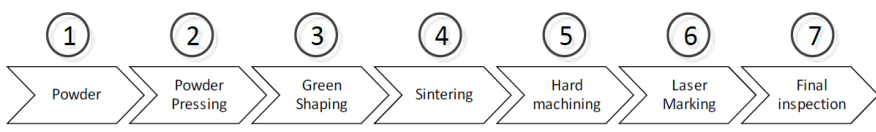

Fig. 1. Manufacturing stages of ceramic hip joint implant components

unstable manner under external loads. Those flaws are introduced to the material mostly during the manufacturing process [6]. Production process of ceramic components (e.g. femoral head implants as considered in this study) consists in seven fundamental steps as shown in Figure 1. Flaws are typically introduced in the first five stages, namely: (1) powder impurities result in inclusions in the final product; (2) inhomogeneous density distribution during pressing results in material voids; (3) chipping and scratching may occur at the green machining stage; (4) gas voids, debondings and cracks may arise during sintering; finally (5) hard machining may result in scratches on the final surface. All of the abovementioned flaws, especially when directly located in stress concentration zones will severely reduce the component's strength. In addition, large scatter in material strength observed experimentally is due to the scatter in size and number of flaws in the material. The intrinsic low resistance against crack formation and growth (the brittleness) is the greatest drawback of ceramic materials in general as it may lead to catastrophic failures without prior warning (e.g. visible plastic deformation) and therefore must be kept under control.

In large series production of ceramic components, processing time is of great importance. Cost effectiveness demands an optimization of manufacturing time. However, at the same time a fully flaw-free production must be guaranteed in order to meet the quality and safety requirements of the medical industry. Therefore, the final nondestructive inspection is inevitable to eliminate all possible faulty components. As mentioned before the most widely applied technique for this purpose is the liquid penetrant testing $[7,8]$. The limiting factors related with the application of this technique, from the economical point of view, include labor intensity, involvement of highly qualified personnel and complex infrastructure required for preparation and cleaning of the samples. This contributes largely to the total production cost. Finally, being all manual process, it is vulnerable to human error, even providing strict controlling policies. Thus, an alternative nondestructive testing method is of great interest to any manufacturer committed to the quality and safety of his products.

The test samples considered for this study are ceramic femoral heads made of zirconia toughened alumina (ZTA) used in total hip arthroplasty (THA). Tested samples had isolated surface breaking cracks introduced into the structure during the manufacturing process. ZTA is a composite ceramic material with zirconia grains $\left(\mathrm{ZrO}_{2}\right)$ embedded in the alumina matrix $\left(\mathrm{Al}_{2} \mathrm{O}_{3}\right)$. The ZTA material is characterized by its excellent mechanical properties, high temperature stability as well as wear and corrosion resistance. The relatively low weight makes ZTA ceramics competitive to metallic materials [13]. In comparison with metals, zirconia toughened alumina share, however, the same drawbacks with most ceramic materials which are: brittleness, susceptibility to cracking that may arise during or due to the manufacturing processes and in the service period. Failures of ceramic components are typically abrupt which makes them hard to detect based on the observation of changes in structural behavior.

Thermographic Nondestructive Testing (TNDT) may provide a viable solution to the problem provided that certain practical factors are met. An important practical factor influencing the effectiveness of TNDT applications relates to the thermal properties of the material under investigation. Thermal diffusivity of a material is a ratio of its thermal conductivity to the specific heat capacity and mass density. Materials with high thermal diffusivity (like aluminum) show fast response for the applied thermal excitation. Their temperature changes quickly, therefore a shorter excitation time and energy levels are sufficient. However, to capture this dynamic thermal response a high framerate, and usually high cost equipment, is required. Low thermal diffusivity materials respond to changes slowly, which means longer inspection times. For low dynamic measurements, however, a lower cost hardware can be used. The ZTA ceramics have thermal diffusivity of approximately $6 \mathrm{~mm}^{2} / \mathrm{s}$ which is much lower than most engineering metals. They are, therefore, well suited for TNDT applications.

The following section presents the two TNDT techniques applied to defect detection in hip joint implants made of ZTA ceramics.

\section{Laser spot thermography}

Laser spot thermography is one of the active TNDT techniques. The energy in this test modality is delivered to the surface of a structure in the form of a laser pulse [14-17]. Energy deposited on a surface is converted into heat and propagates through the material in form of thermal waves that propagate radially, away from the excitation location. The temperature profile changes over time, which can be measured with an infrared (IR) camera. In a thermally isotropic medium, generated heat diffuses radially from the excitation point in a symmetrical manner and the observed temperature decays over the time. The symmetric temperature profile can be, however, distorted by the presence of thermal barriers. These include cracks, voids or other features with low thermal conductivity that change the path of thermal wave propagation. It is worth mentioning that a very similar approach, flying spot thermography, is also used. In this case the energy from a laser source is delivered continuously and laser spot moves over the measured surface [18-21]. Laser spot thermography is a fully noncontact test method which makes it very attractive for all applications where sterile environment is desirable e.g. manufacturing of electronic or medical products. As any other measurement technique, also laser spot thermography has certain drawbacks. These include a high initial cost of the measurement system (mainly the laser source and thermal 
camera), the ability to reveal mostly the surficial damage and in some cases the necessity of surface treatment for very glossy surfaces in order to improve thermal emissivity.

The hardware arrangements for laser spot thermography are relatively simple and consist in an IR camera, pulsed laser source with optical head, positioning system and PC computer for measurement control and data acquisition. In order to obtain high spatial resolution and increase the detection sensitivity to small damage, it is preferable to position the IR camera close to the measured surface. In addition, the analysis is possible only in the vicinity of excitation laser spot, where thermal gradient is high. This limits the effective measurement Field of View (FOV). Therefore, assuming that we do not want to move the test sample, there are at least two measurement scenarios that can be applied in practice to cover larger measurement fields and retain high accuracy of measurement. The first possibility is illustrated schematically in Figure 2. In this setup, the IR camera location is fixed with respect to the test sample while the optical head of the excitation laser is moving. As a result, the analyzed FOV is fixed and laser spot is moving illuminating different points on the sample. The second possibility is illustrated in Figure 3. In this case, both IR camera and optical head are moving simultaneously with respect to the test sample. As a result, the FOV changes with each measurement point but the location of laser spot in the thermal image is constant. The choice of a particular test setup determines, to some extent, the subsequent signal processing stage.

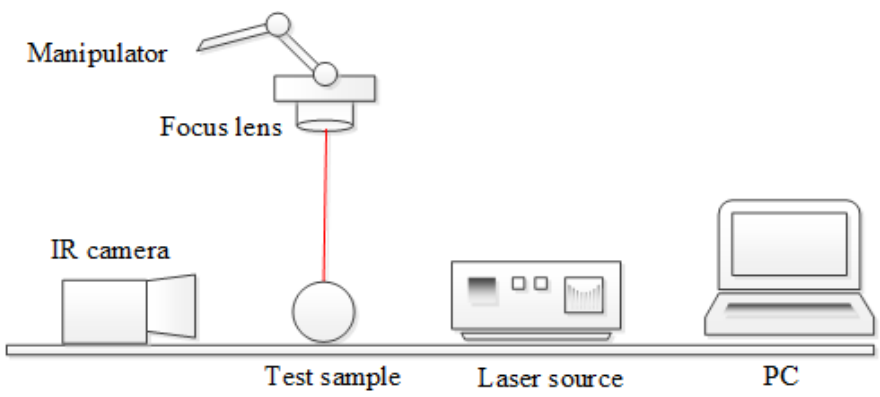

Fig. 2. Laser spot thermography test setup - fixed camera

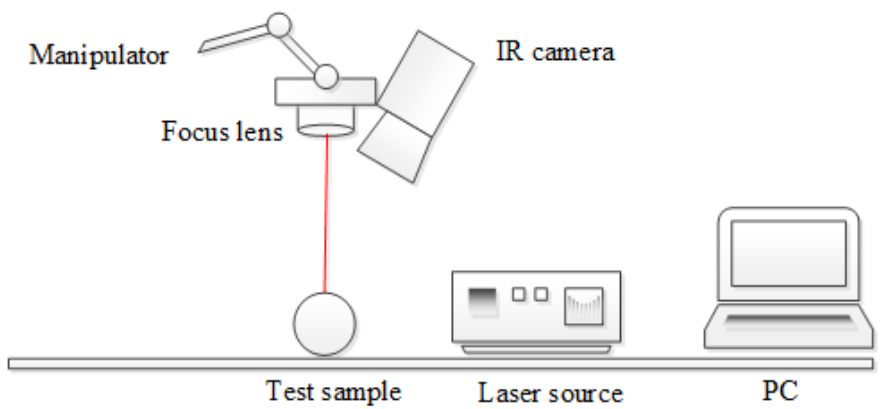

Fig. 3. Laser spot thermography test setup - moving camera

For both test scenarios, the measurement procedure consists of an acquisition of many individual measurements performed in a point-by-point manner. A thermal sequence is acquired and stored for every excitation point. The post processing, in our current deployment of laser spot thermography, is performed offline after the measurements for all excitation locations are acquired. All thermographic sequences need to be merged and evaluated together to obtain the diagnostic information from a complete dataset. In order to do this, a proper synchronization between an IR camera, laser controller and manipulator moving the optical head needs to be maintained. The post processing algorithms in this case are more complex as has been described in [14-16].

In this study, the experimental setup shown schematically in Figure 4 was adopted. The measurement system consists in: (1) a cooled InSb photon detector IR camera FLIR Silver; (2) a LIMO diode laser source operating at $808 \mathrm{~nm}$ wavelength with focusing optics; (3) a 2D Cartesian manipulator and (4) a PC computer with measurement and data acquisition software. The IR camera was equipped with a $27 \mathrm{~mm}$ lens $\left(16^{\circ} \times 20^{\circ}\right.$ view angles) that was placed approximately $160 \mathrm{~mm}$ away from measurement object. The image was focused on a plain containing the nearest point of the measured surface and remained unchanged over the whole measurement sequence. Given a high depth of field (DOF) of the IR camera optics the whole test sample remained in focus. The laser optical head was equipped with a spherical focusing lens with focal length equal to $120 \mathrm{~mm}$. Changes in the focal spot diameter were obtained by changing the distance of the optical head from the tested surface. The laser beam was pointed onto the measured test sample vertically from the top, and could be shifted along a straight line with a motorized linear stage. Therefore, due to curvature of the tested object, the laser spot shape on the surface was slightly elliptical, rather than circular. For the same reason, the laser spot location did move linearly in the FOV of the IR camera, which was positioned from a side to the measured sample, as shown in Figure 4. This was the limitation of the available measurement setup. The acquisition of the thermal sequences was triggered by the TTL (Transistor-Transistor Logic) signal provided by the laser controller. In addition, the IR camera had a defined pre-trigger time of $2000 \mathrm{~ms}$ to store thermal images in that interval preceding the laser pulse that could be used for background subtraction. All measurements were performed with the $120 \mathrm{~Hz}$ frame rate of the IR camera and pixel resolution of $320 \times 256$.

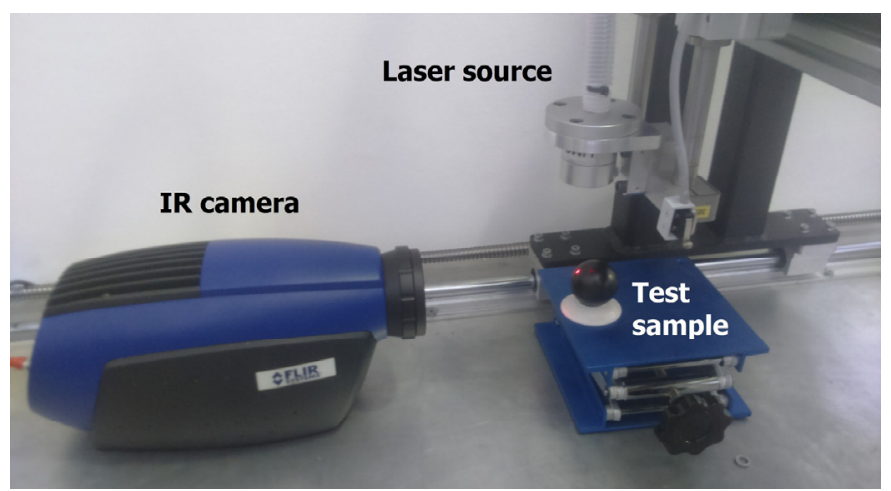

Fig. 4. Experimental setup adopted for laser spot thermography tests 
The test sample was a femoral head implant made of ZTA ceramics. The diameter of the implant was equal to $40 \mathrm{~mm}$ and it contained a barely visible surface-breaking crack with a total length of approximately $14 \mathrm{~mm}$. For testing, the sample was painted in black in order to equalize and increase its thermal emissivity. Before performing the target measurement, a series of pretest experiments was performed in order to find the best excitation settings. The following ranges of laser parameters were considered: laser power 10-30 W, pulse duration 50-500 $\mathrm{ms}$ and spot diameter equal to $1 \mathrm{~mm}$, which was the lowest value possible to obtain with the optical head. The excitation points were evenly distributed over the test area and allowed to reveal the location of crack in the sample. These preliminary tests allowed to empirically select the laser source settings for which the best results were obtained. The target measurements were performed for the following laser source parameters: laser power $20 \mathrm{~W}$, pulse duration $100 \mathrm{~ms}$ and laser spot diameter of $1 \mathrm{~mm}$. Figure 5 shows the positions of five excitation points located in the vicinity of surface braking crack in the sample identified roughly in the pretest stage. The points were obtained by moving the optical head along a straight line with a linear stage. However, due to the curvature of the sample the points were located on a curve in the measurement FOV as shown schematically in Figure 5.

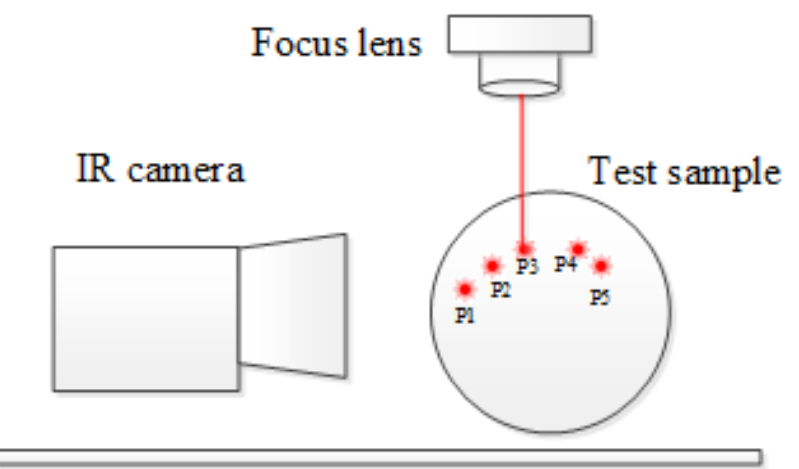

Fig. 5. Locations of excitation points in laser spot thermography test
The final outcome from laser spot thermography test was obtained with use of a semi-automated post processing algorithm, shown in Figure 7, which has been introduced in [16]. Thermographic sequences acquired for all excitation locations needed to be merged into a single sequence and processed to identify possible damages.

The processing starts with an automatic subroutine ('PTW loader') importing all constituent thermal sequences from separate input files recorded for each excitation point. Subsequently, preprocessing is performed starting with background temperature subtraction for each sequence. Background temperature is calculated as an average of the first five acquired frames, prior to laser excitation. All sequences are then added together and equalized if necessary. Figure 7 presents and exemplary sequence of thermal images for points from P1 to P5 (as shown in Figure 5) and the resultant merged sequence. In the next step, an image frame is selected corresponding to the mid-time of the cooling phase (i.e. the mid-time between the peak temperature occurring at location of laser spot and a time when the cooling curve reaches a plateau). The user can, however, change this time to perform the analysis at different time instances if necessary. Then automatic edge detection procedure based on the Roberts filter is performed. In the current application, the parameters of the filter were chosen empirically. Median filtering is applied in the next step to remove the salt and pepper noise. Edges identified in the thermal image correspond to thermal barriers caused by defects or structural features. Therefore, in order to facilitate data interpretation, identified edges are overlaid on the on the first recorded thermal image to obtain the final result as shown in Figure 8.

Figure 8 shows the final outcome of the measurement which consists of a detected crack (red curve) overlaid on the thermal image of the sample obtained before excitation. The postprocessing was performed based on thermographic sequences obtained for points P1 to P5 (as shown in Figure 5). Both crack location and size are clearly visible and can be precisely measured knowing the geometrical calibration of the IR camera.

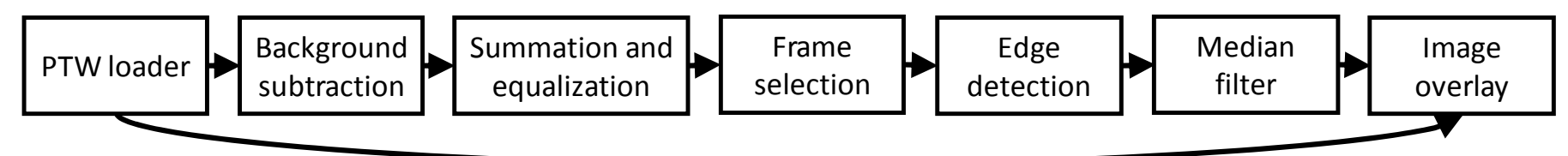

Fig. 6. Block diagram of thermal image processing algorithm for laser spot thermography

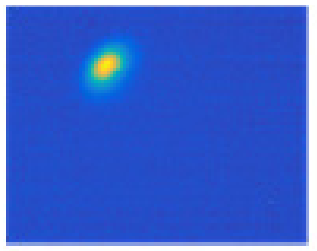

P1

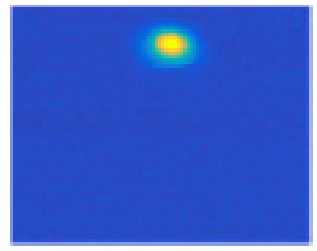

P5

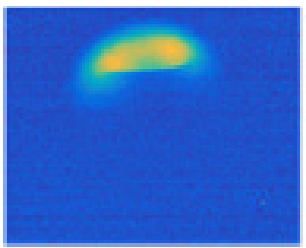

sum

Fig. 7. Laser spot thermography - intermediate experimental results 


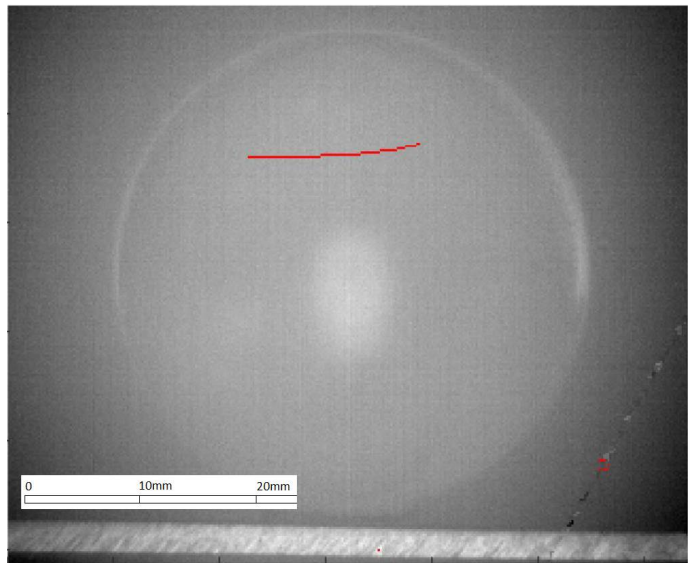

Fig. 8. The final outcome of laser spot thermography test. Red curve indicates the location and extent of surface breaking crack

\section{Vibrothermography}

Vibrothermography is another active TNDT technique [2224]. In this case, the energy is delivered to the tested object in the form of elastic waves generated by piezoceramic transducer assembly. Typically, elastic waves in the frequency range from $15 \mathrm{kHz}$ to $70 \mathrm{kHz}$ are introduced to the material in a contact manner. The waves propagate in the material, and when they encounter a discontinuity (such as a crack) part of the elastic energy is dissipated into heat, primarily through friction [24-25]. Generated heat propagates to the surface where it can be detected by an IR camera.

Vibrothermography is a robust test method with a simple test setup, as shown schematically in Figure 9. The measurement procedure usually takes no longer than a few seconds, as initial calibration and complex signal processing can be avoided. Vibrothermography is a dark-field thermographic method as the source of heat is the discontinuity itself and the healthy portion of the sample does not dissipate heat on a comparable level. The method requires contact ultrasonic excitation and, in a correct test setup, the vibration source and the test object should remain in full contact during the whole excitation cycle. This is assured by applying a normal force pressing the vibration source against the excited surface, which assures safe and efficient transfer of vibrations into the test sample. In our case, this is realized by an adjustable compressive spring system (green column in Figure 10), but often it is done by a pneumatic actuator. If clamping force of the vibration source is to low, the hammering effect may occur, which may cause surface damage of the test sample. Often, spacers of different materials are applied between the vibration

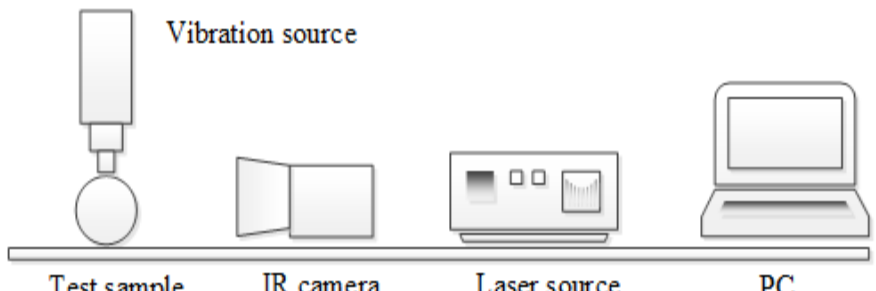

Fig. 9. Vibrothermography test setup source and the test sample to prevent damage. In the analyzed case, a Teflon spacer was used for this purpose.

Figure 10 shows the experimental test rig used for vibrothermographic testing in this study. The system consists in a vibration source (a $35 \mathrm{kHz}$ bolt-clamped Langevin type resonator with waveguide), a photon detector IR camera FLIR Silver, ultrasonic amplifier and a PC with control and data acquisition software.

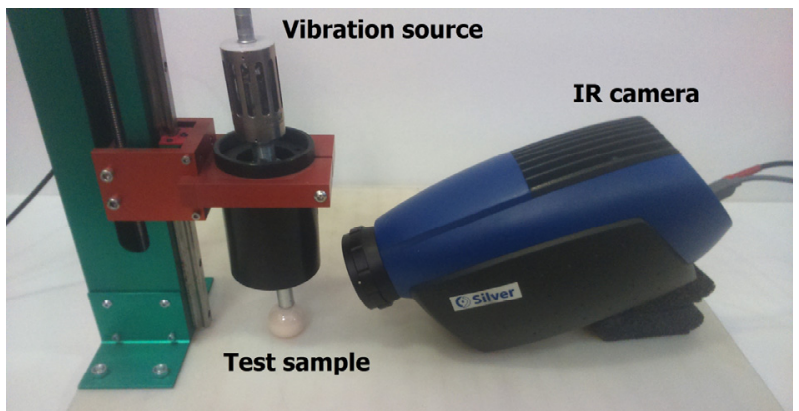

Fig. 10. Experimental setup adopted for vibrothermography tests

Experimental tests were performed using the following parameters: excitation time equal to $1 \mathrm{~s}$, vibration source operating at $35 \mathrm{kHz}$ and $500 \mathrm{~W}$ input electric power. The infrared camera acquired sequences of thermal images for 4 seconds with $120 \mathrm{~Hz}$ frame rate.

Figure 11 shows thermal response of the test sample during vibration excitation. Figure 12 shows temperature time history for two points: one was located in the crack area (P1) while the other was located away from the crack area (P2). The temperature rise on damage is in the order of $0.3^{\circ} \mathrm{C}$. Further signal post processing was applied to identify the existing surface braking crack unequivocally.

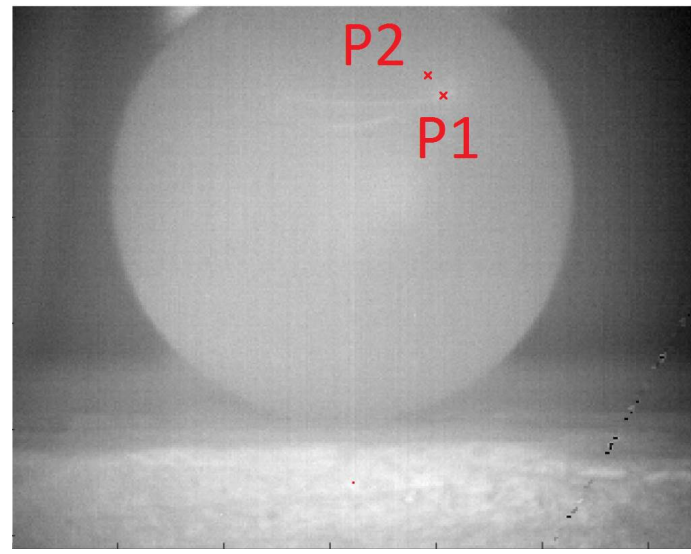

Fig. 11. Field Of View (FOV) of the thermographic camera

Figure 13 presents a single frame from the acquired thermal sequence (at time equal to $1500 \mathrm{~ms}$ ) from which the background temperature distribution was subtracted. The frame was selected by identifying the largest temperature change over all pixels in the thermal sequence. The presence and location of the crack can be already identified, but in order to facilitate interpretation of the result, further image processing was applied. For the thermal 


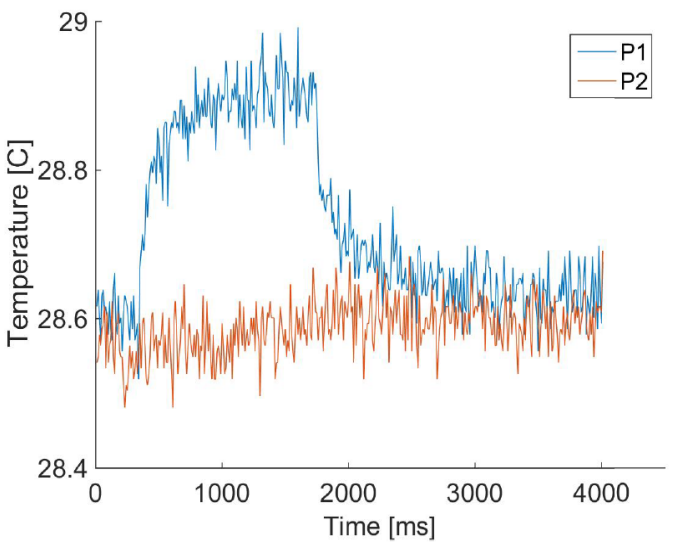

Fig. 12. Temperature time history plots at points P1 (blue curve) and P2 (orange curve) as marked in Fig. 10

image shown in Figure 13, an edge detection algorithm (Matlab implementation based on Roberts filter) was used to extract the crack signature. Subsequently, detected edges were overlaid on the background thermal image. The result is shown in Figure 14. It can be seen that a more pronounced heating occurs on the right side of the crack which may suggest that the crack opening was not uniform over the crack lengt.

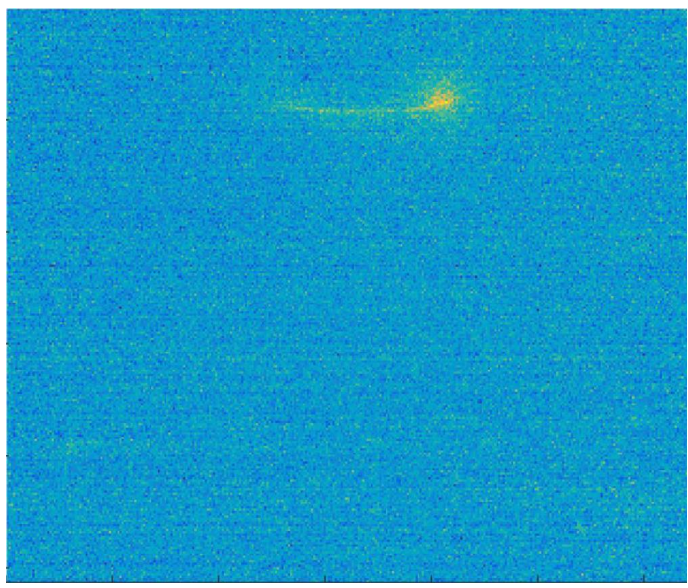

Fig. 13. Thermal image acquired during the vibrothermographic test at time equal to $1500 \mathrm{~ms}$ after the start of ultrasonic excitation

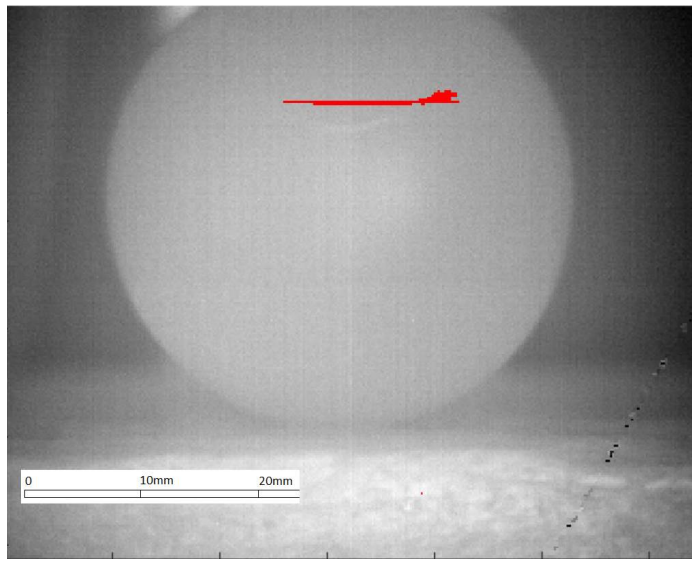

Fig. 14. The final outcome of vibrothermographic test. Red curve indicates the location and extent of surface breaking crack

\section{Conclusions}

The paper presented a feasibility study for the application of laser spot thermography for nondestructive testing of surface breaking cracks in zirconia toughened alumina ceramic components. Experimental results have been compared with vibrothermographic test performed on the same test specimen. The location and size of the crack were correctly revealed by both techniques as shown in Figure 8 and Figure 14 respectively. It has been verified experimentally that both considered measurement techniques can be effectively used to detect surface breaking cracks in ceramic components, with comparable experimental effort.

The advantage of laser spot thermography over vibrothermography is a fully non-contact nature of the measurement. Both excitation and measurement are done from a distance using a pulsed laser source and an IR camera, respectively. This may be beneficial for certain applications, like the considered case of testing medical implants, where contact test methods may be not recommended or even prohibited. Moreover, one could imagine a scenario where laser spot thermography measurement is automated (for both signal acquisition and signal processing stages) in order to provide an efficient end of line test method for medical products. The main limitation of laser spot thermography is the fact that it is capable of identifying mostly surficial damage on areas exposed to the view of an IR camera. Therefore, cracks buried in the volume of a material or at the bottom of convex geometrical features may be difficult or impossible to detect. This is, however, also true for some other techniques like visual inspection or liquid penetrant testing that are currently used to identify cracks in medical implants. Therefore, laser spot thermography could be considered as an alternative to these techniques, rather than for approaches capable of detecting damage also in the volume of a material like x-ray or ultrasound.

Further research is planned in this area to characterize the critical detection parameters in ceramic components like the minimum detectable defect size and further automate the diagnostic process.

\section{REFERENCES}

[1] P.O. Moore, Nondestructive Testing Handbook, Vol 10, Overview, $3^{\text {rd }}$ ed., American Society for Nondestructive Testing (2012).

[2] T. Stepinski, T. Uhl, W. Staszewski, Advanced Structural Damage Detection: From theory to Engineering Applications, Wiley (2013).

[3] X. Maldague X, Theory and Practice of Infrared Technology for Nondestructive Testing, Wiley (2001).

[4] R. Bradt, D. Hasselman, D. Munz, V. Sakai, Y. Shevchenko, Fracture Mechanics of Ceramics, Springer (2012).

[5] B. Perrichon, H. Liu, J. Chayalier, L. Gremillard, B. Reynard, F. Farizon, J. Liao, J. Geringer, Ageing Shocks and War Mechanism in ZTA and the Long-Term Performance of Hip Joint Materials, J. of Mater. (2017). 
[6] D. Munz, T. Fetts, Ceramics: mechanical properties, failure behavior, material selection, Springer Science (2013).

[7] British Institute of Non-Destructive Testing, Liquid penetrant inspections, The British Institute of Non-Destructive testing (2017).

[8] Magnaflux, Zyglo Fluorescent Penetrant Operating Instructions for Bulk Applications, NDT Supplies (2017).

[9] M. Krishnapillai, R. Jones, I.H. Marshall, M. Bannister, N. Rajic, Thermography as a tool for damage assessment, Comp. Struct. 67 (2005).

[10] T. Sakagami, S. Kubo, Applications of Pulse Heating Thermography and Lock-in Thermography to Quantitative Nondestructive Evaluations, Infr. Phys. and Tech. (2002).

[11] G. Pitarresi, Lock-In Signal Post-Processing Techniques in Infra-Red Thermography for Materials Structural Evaluation, Exp. Mech. 55 (2015).

[12] J. Roemer, L. Pieczonka, M. Szwedo, T. Uhl, W. Staszewski, Thermography of Metallic and Composite Structures - review of applications, The e-Journal of Nondestr. Test. (2013).

[13] STC Superior Technical Ceramics, Zirconia-Toughened Alumina, White paper (2014).

[14] T. Li, D. Almond, D. Andres, S. Rees, Crack Imaging by Scanning Pulsed Laser Spot Thermography, NDT\&E Int. 44 (2), (2010).

[15] S. Burrowsm, A. Rashed, D. Almondm, S. Dixon, Combined Laser Spot Imaging Thermography and Ultrasonic Measurements for Crack Detection. Nondestr. Test. and Eval. (2007).

[16] J. Roemer, L. Pieczonka, T. Uhl, Laser Spot Thermography of Welded Joints. Diagnostyka 15 (2014).
[17] J. Roemer, L. Pieczonka, T. Uhl, Laser Spot Thermography for Crack Detection in Aluminium Structures, $7^{\text {th }}$ Int. Symp. on NDT in Aerospace (2015).

[18] C. Hermosilla-Lara, P. Joubert, D. Plancko, Identification of Physical Effects in Flying spot Photothermal Non-Destructive Testing, Eur. J. Appl. Phys. (2003).

[19] N. Montinaro, D. Cerniglia, G. Pitarresi, Flying Laser Spot Thermography technique for the NDE of Fibre Metal Laminates disbonds., Compos. Struct. (2017)

[20] A. Yun-Kyu, K. Ji, S. Hoon, Laser Lock-in Thermography for Fatigue Crack Detection, NDT \& E Int. 65 (2014).

[21] T. Almond, D. Rees, Crack Imaging by Scanning Laser-line Thermography and Laser Spot Thermography, Meas. Sci. and Tech. (2011).

[22] K. Reifsnider, E. Henneke, W. Stinchcomb, The Mechanics of Vibrothermography, in: W. Stinchcomb, C. Duke, E. Henneke, K. Reifsinder (Eds.), Mechanics of Nondestructive Testing, Springer (1980).

[23] L. Pieczonka, M. Szwedo, Vibrothermography, in: T. Stepinski, T. Uhl, T. Staszewski(Eds.), Advanced Structural Damage Detection: From Theory to Engineering Applications, 233-61, Wiley (2013).

[24] L. Pieczonka, F. Aymerich, G. Brozek, M. Szwedo, W.J. Staszewski, T. Uhl, Modelling and numerical simulations of vibrothermography for impact damage detection in composites structures, Struct. Control. Heal. Monit. 20 (4), (2013).

[25] C. Homma, M. Rothenfusser, J. Bauman, R. Shannon, Study of the Heat Generation Mechanism in Acoustic Thermography, Quant. Nondestr. Eval., AIP Conference Proceedings 820 (2006). 